\title{
Physiology of Non-Voltage-Gated Ion Channels
}

\author{
Rana Toghan ${ }^{1}$ and Khaled A. Abdel-Sater ${ }^{2 *}$ \\ ${ }^{1}$ Physiology Department, Qena Faculty of Medicine - South Valley University \\ ${ }^{2}$ Physiology Department, Faculty of Medicine - Al-Azhar University \\ *Corresponding Author \\ Department of Physiology, \\ Faculty of Medicine, Al-Azhar University \\ Egypt \\ Tel:00201067970804 \\ E-mail:Khaled71111@yahoo.com
}

Received:20 September 2020;| Revised:07 September 2021; | Accepted:13 December 2021

\begin{abstract}
Ion channels are transmembrane proteins that allow ions to flow across the cell membrane. They are characterized by selectivity. Physiological processes include excitation-contraction coupling, synaptic transmission, hormone secretion, and sensory transduction.

Ion channels can be classified according to the presence or absence of gates; open and gated channels. Members of gated channels are; voltage-gated, ligand-gated, and other gating mechanisms. Ion channels that open following a change in the membrane voltage potential are known as voltage-gated ion channels. Ligand-gated ion channels allow ions to flow across the pore in response to the binding of an external chemical messenger (ligand). They are divided into four families: cys-loop receptors, ionotropic glutamate receptors, ATP-gated $\mathrm{P}_{2} \mathrm{X}$ receptors, and ligand-gated $\mathrm{Ca}^{2+}$ channels. Other gating includes activation/inactivation by second messengers from within of the cell membrane. They include; transient receptor potential, cyclic nucleotide-gated channels, ATP sensitive $\mathrm{K}+$ channels, calcium-activated $\mathrm{K}^{+}$ channels, mechanosensitive ion channels, acid-sensing ion channels, and cystic fibrosis transmembrane conductance regulator $\mathrm{Cl}^{-}$channels. This review aims to show the physiological bases of ion channel functions. That is important in understanding the pathophysiology, and treatment of channelopathies.
\end{abstract}

Keywords: Ion Channels, Voltage-Gated, Ligand-Gated, $\mathrm{Na}^{+}$Channels, $\mathrm{K}^{+}$Channels, $\mathrm{Ca}^{2+} \mathrm{Channels}$

\section{Introduction}

The flow of inorganic ions, like $\mathrm{Na}+, \mathrm{K}+, \mathrm{Ca}^{2+}$, or $\mathrm{Cl}$ - from one side of the cell membrane to the other occurs through the ion channels ${ }^{[1]}$. The speed of ion diffusion through the channel is extremely high. They allow only ions of a specific size and/or charge to pass ${ }^{[2]}$. Ion channels play a fundamental role in physiological processes, such as excitation-contraction coupling, nutrient transport, hormone secretion, neurotransmitter release, processing of data within the brain, and output from the brain to peripheral tissues ${ }^{[3]}$. They also play a task in various diseases and disorders, for example; myasthenia, epilepsy, ataxia, Alzheimer's disease, migraine, schizophrenia, 
and polycystic kidney [4]. Therefore, ion channels are very important as therapeutic targets. Our understanding of the physiology of ion channels is going to be essential in study new agents to spot novel therapeutic targets for the patient benefit [2].

Ion channels are often classified in several distinct ways. They'll be classified by types of ions, gating, and localization of proteins. Also, they're going to be classified by their electrophysiological properties and by their pharmacological sensitivity to toxins and medicines. According to the presence or absence of gate, ion channels are classified into open (leak or simple) channels, and gated channels; they're closed during rest by protein act as a gate. In most cases, the gate opens in response to a selected stimulus. The most important stimuli that are known to cause ion channels to open are a change within the voltage across the membrane (voltage-gated channels) or the binding of a ligand (ligand-gated channels). The ligands are often either an extracellular mediatorspecifically, a neurotransmitter (ligand-gated channels)-or an intracellular mediator, like an ion or a nucleotide (other-gated channels) ${ }^{[5]}$.

\section{Ligand-gated channels (lgics)}

Ligand-gated ion channels are integral membrane proteins that are opened, or gated, by the binding of an extracellular neurotransmitter or hormone to a selected receptor ${ }^{[6]}$.

Members of ligand-gated ion channels are often divided into four families: (I) cys-loop receptors, (II) ionotropic glutamate receptors, and (III) ATP-gated $\mathrm{P}_{2} \mathrm{X}$ receptors (IV) ligand-gated $\mathrm{Ca}^{2+}$ channels ${ }^{[7]}$.

\subsection{Cys-loop Receptors (Pentameric Ligand- Gated Ion Channels (pLGICs)}

They include nicotinic acetylcholine (nACh), serotonin (5-HT3), and zinc activated receptors (ZAC) that conduct cations, also as GABAA and glycine (Gly) receptors which conduct anions ${ }^{[8]}$.

\subsubsection{Nicotinic acetylcholine receptors}

Ligands may be endogenous like acetylcholine, choline or exogenous like nicotine and toxic alkaloid epibatidine obtained from poison dart frogs.
The binding of the ligand induces conformational change and leads to an influx of cations like $\mathrm{Na}+$, $\mathrm{K}^{+}$, and $\mathrm{Ca}^{2+}{ }^{[7]}$. They're found within the peripheral nervous system, primarily at the myoneural junction and ganglion. NM and NN are the 2 sorts of nicotinic acetylcholine receptors; $\mathrm{NM}$ is found within the neuro-muscular junction while NN is found in a ganglion. Due to the opening of the acetylcholine-gated channels in the endplate membrane, a large number of $\mathrm{Na}+$ ions from the ECF enter inside the muscle fiber causing a local positive potential change inside the muscle fiber membrane called the endplate potential. The endplate potential is non-propagative but when a critical level of $-60 \mathrm{mV}$ is reached, it triggers the event of AP within the muscle cell. Voltagedependent $\mathrm{Na}^{+}$channels at the adjacent sites are open, allowing more $\mathrm{Na}^{+}$in. The produced APs spread along the surface of the striated muscle membrane. This is often detected by $\mathrm{Ca}^{2+}$ channels within the T-tubules of the muscle which respond by opening and allowing a $\mathrm{Ca}^{2+}$ ion influx leading to contraction ${ }^{[9]}$.

Abnormal nAChRs function, as in congenital myasthenia, means there is insufficient activation of the $\mathrm{Ca}^{2+}$ channels resulting in reduced contraction. A prominent feature of NN receptors is their high permeability to $\mathrm{Ca}^{2+}$. Many of the $\mathrm{NN}$ receptors within the brain are located presynaptically on glutamate-secreting axon terminals, and that they facilitate the discharge of this neurotransmitter. They're important in cognitive functions and behavior ${ }^{[10]}$.

\subsubsection{5-Hydroxytryptamine 3 (5-HT3) receptor channels}

Serotonin (5-hydroxytryptamine; 5-HT) is present within the highest concentration in blood platelets and within the alimentary canal, where it's found in the enterochromaffin cells and therefore the plexus myentericus. It's also found in the midline raphe nuclei of the brainstem, which project to a wide portion of the CNS including the hypothalamus, visceral brain, neocortex, cerebellum, and medulla spinalis. There are seven main kinds of 5-HT receptors but only the $5-\mathrm{HT}_{3}$ is ligandgated. Its pore is permeable to $\mathrm{K}^{+}$and $\mathrm{Na}^{+}$ions with some subtypes also showing selective $\mathrm{Ca}^{2+}$ ion conductance. They're expressed within the central 
and peripheral nervous systems also because of the gastric-intestinal tract ${ }^{[11]}$.

Radiotherapy

and

chemotherapeutics, like cisplatin and doxorubicin, can cause nausea and vomiting because they induce 5-HT to be released within the alimentary canal. Consequently, 5- $\mathrm{HT}_{3}$ receptors located on vagal nerve terminals are stimulated resulting in activation of the vomiting center within the brain. Setrons are antiemetics that inhibit 5-HT3 receptor activity and are wont to to counter nausea and vomiting related to these treatments for cancer. 5$\mathrm{HT}_{3}$ receptors have also been related to gastric reflux disease where the acid content of the stomach slowly erodes the liner of the esophagus ${ }^{[12]}$.

In the central nervous system pre-synaptic 5$\mathrm{HT}_{3}$ receptors are involved within the control of neurotransmitter release like substance $P$, with agonists enhancing dopamine and GABA release. They also mediate inflammation and chronic pain. Additionally, to the emetic effect, stimulation of $5-\mathrm{HT}_{3}$ receptors increases anxiety levels ${ }^{[7]}$.

\subsubsection{Zinc activated channel receptors (ZAC)}

Zinc is the second most abundant chemical element (after iron) essential for all living organisms. It's involved during a sort of biological process, as a structural, catalytic, and intracellular and intercellular signaling component ${ }^{[7]}$. ZAC is present within the human, chimpanzee, dog, cow and opossum genomes, but is functionally absent from mouse, or rats. In humans, it founds in prostate, thyroid, trachea, lung, brain (adult and fetal), medulla spinalis , striated muscle, heart, placenta, pancreas, liver, kidney, and stomach. It's voltage-independent and exhibits spontaneous activity ${ }^{[12]}$.

Not much is understood about the pharmacology or physiological function of those receptors. They're cation channels that are activated by $\mathrm{Zn} 2+$ ions protons and copper and inhibited by $(+)$-tubocurarine, a nicotinic receptor antagonist ${ }^{[13]}$.

\subsubsection{GABA Receptors}

Gamma-aminobutyric acid (GABA) is usually accepted because of the major inhibitory neurotransmitter within the vertebrate brain. Its receptor has been found more extensively throughout the nervous system within the thalamus (specifically the reticular nucleus), hypothalamus, cerebellum, basal ganglia, and hippocampus. GABA receptors within these areas function as feedback loops [7]. Instance, within the cerebellum, Mossy fibers stimulate deep nuclei and granular cells. Then granular cells exit (by glutamate) the Purkinje, basket stellate cells, and Golgi. Purkinje cells are inhibitory (by GABA) to deep nuclei. The Golgi cells inhibit the granular cells. Cortico-Ponto-Mossy fiber stimulates the deep nuclei of the cerebellum which discharge excitatory to activate signals that help in the initiation of movement. At the top of the movement, Purkinje cells would are excited by granular cells so, they send inhibitory to deep nuclei (turn off) which stop the movement (= negative feed-forward inhibition) loops ${ }^{[14]}$.

There are two closed circuits between the cerebral cortex and basal ganglia. The first one is a direct pathway and starts at the striatum to the globus pallidus internal then to the thalamus. It's an excitatory pathway because the striatal neurons secrete GABA which inhibits GABA release from globus pallidus interna that causes stimulation of the thalamus. And it facilitates voluntary movement. The $2^{\text {nd }}$ pathway is indirect and starts at striatum to globus pallidus externa to subthalamus to globus pallidus interna then to thalamus). This pathway is inhibitory because the striatal neurons secrete GABA which inhibits GABA release from globus pallidus externa, therefore the nucleus is released from inhibition and secretes glutamate which stimulates GABA to release from globus pallidus interna that causes inhibition of the thalamic cortical stimulation. This pathway acts as an opportunity to prevent the movement. Also, it prevents spontaneous useless involuntary movements initiated by the cortex ${ }^{[15]}$.

Also normally, the striatum is connected to the substantia nigra reticulares by inhibitory neurons that secrete the inhibitory transmitter GABA at their termini. In turn, cells of the substantia nigra send inhibitory dopaminergic neurons back to the striatum, to inhibit its acetylcholine. On the other hand, dopaminergic neurons arise from substantia nigra parsa compacta to the striatum, where they excite the direct pathway and inhibit the indirect 
pathway. The net result of both is stimulation of the thalamus ${ }^{[16]}$.

Parkinson's, Huntington and Alzheimer's diseases are examples of GABA disorders. GABA receptors are documented as the main drug target for several classes of drugs to achieve sedative, hypnotic, anesthetic, and anticonvulsive effects ${ }^{[17]}$.

\subsubsection{Glycine Receptors}

Glycine receptors are also present within the central nervous system especially within the spinal cord, the brainstem and the retina, where they mediate synaptic inhibitory signaling in sensory and motor pathways ${ }^{[7]}$.

Hyperekplexia and Autism are samples of glycine receptors disorders. Though there are not any specific pharmacologic agents that focus on glycine receptors yet, it's pertinent to say the consequences of strychnine poisoning. Used as a pesticide, if one experienced high exposure this might end in effects associated with glycine receptor antagonism. Symptoms include Cawake seizures described as painful muscle contractions while the patient is conscious - the clinical course lacking a post-ictal period normally seen in seizure activity ${ }^{[18]}$.

\section{Ionotropic Glutamate Receptors (iGluRs)}

Glutamate is the principal excitatory neurotransmitter within the central nervous system and is thus critical for sensory and cognitive functions. They play a key role in synaptic plasticity-future potentiation, which is significant for learning and short-term memory. They are also involved in nociception. This receptor is split into four subtypes supported their ligand-binding properties or pharmacology and sequence similarity: AMPA ( a -amino-3-hydroxy5- methyl-4-isoxazole propionic acid) (Glu A1 - 4), kainate (KARs; Glu K1 - 5), NMDAN (methyl-daspartate) (Glu N1, Glu N2A - D and Glu N3A-B), and delta (Glu D1 and Glu D2) receptors ${ }^{[7]}$.

Long term potentiation means rapidly developing persisting stimulation of postsynaptic neuron due to repeated stimulation of the presynaptic neuron (mainly in the hippocampus). Repeated stimulation leads to continual $\mathrm{Na}+$ influx and persistent depolarization. AMPA receptor is
$\mathrm{Na}^{+}$dependent while, NMDA is $\mathrm{Ca} 2+$ dependent ${ }^{[19]}$. $\mathrm{Ca}^{2+}$ cannot move through these channels because they are blocked by magnesium ions. The persistent depolarization (more than $20 \mathrm{mv}$ ) by $\mathrm{Na}$ exerts a repulsive force on the magnesium ions and ca influx that will activate protein kinases. This leads to glutamate receptor channels become more sensitive to glutamate, and it causes (by nitric oxide) the presynaptic cell to release more glutamate. The net result is larger and more prolonged EPSPs ${ }^{[20]}$.

Excessive activation of NMDARs followed by $\mathrm{Ca}^{2+}$-load results in necrobiosis in cerebral insult in conditions, like stroke or neurodegenerative conditions, including Alzheimer's disease or Huntington's disease. This is often while AMPARs are linked to many neurodevelopmental disorders, including schizophrenia, Alzheimer's and Parkinson's diseases, and a contributing factor to the proliferation of glioblastoma tumors. It's also believed that AMPARs play a task in depression, seizure spread, and neuronal damage linked with epilepsy ${ }^{[2]}$.

\section{ATP-Gated P2X Receptors}

The ATP-gated P2X receptors are nonselective cation channels that are activated by extracellular adenosine 5 triphosphate (ATP) permeable to $\mathrm{Na}^{+}$, $\mathrm{K}^{+}$, and $\mathrm{Ca}^{2+}$. They're expressed in the peripheral and central nervous systems and have distinct properties as ligand-gated ion channels due to their activation by extracellular ATP, which is an excitatory neurotransmitter released from sympathetic, sensory and enteric nerves ${ }^{[21]}$.

They're involved in various physiological functions like muscle contraction, neurotransmitter release and immune responses. It is suggested that abnormal pain signaling through $\mathrm{P}_{2} \mathrm{X}$ receptors contributes to neuronal sensitization and chronic pain. $\mathrm{P}_{2} \mathrm{X}$ receptors are expressed in glia cells and seem to contribute to neuroinflammation and chronic pain. $\mathrm{P}_{2} \mathrm{X}$ is a potential drug target in neurological diseases and disorders, such as neuropathic pain and epilepsy ${ }^{[2]}$.

\section{Ligand-gated $\mathrm{Ca}^{2+}$ channels}

\subsection{IP3 receptors (IP3R)}

It plays an important physiological role in cellular division, cell proliferation, apoptosis, 
fertilization, development, behavior, learning, and memory. When cells receive signals through receptors on the cell wall, this activates $\mathrm{Gq}$ protein that activates phospholipase $\mathrm{C}$ which split the membrane phospholipid (phosphatidylinositol diphosphate) into IP3 and diacylglycerol. IP3 binds to the IP3 receptor and releases $\mathrm{Ca}^{2++}$. Diacylglycerol and $\mathrm{Ca}^{2+}$ diacylglycerol activate the protein kinase $\mathrm{C}$ enzyme causes the response ${ }^{[4]}$.

IP3 was found not only to release $\mathrm{Ca}^{2+}$, but also to release IRBIT (IP3 receptor-binding protein released with inositol trisphosphate) essential for the regulation of acid-base equilibrium, RNA synthesis, and ribonucleotide reductase ${ }^{[22]}$.

The dysregulation of the IP3 receptor causes various sorts of diseases in human-like ataxia, vulnerability to neuronal degeneration, heart condition, exocrine secretion deficit, and taste deficit ${ }^{[23]}$.

\subsection{Ryanodine $\mathrm{Ca}^{2+}$ receptors $(\mathrm{RyR})$}

It is important in neurotransmitter release and muscle contraction. When activated secondary to dihydropyridine receptors activation the RyR, releases $\mathrm{Ca}^{2+}$ from the sarcoplasmic reticulum or endoplasmic reticulum, an essential step for contraction of skeletal, heart, and smooth muscle. In the heart, $\mathrm{Ca}^{2+}$ release is initiated by the $\mathrm{Ca}^{2+}$ influx through the fast-activating $\mathrm{T}$-type $\mathrm{Ca}^{2+}$ channel and maintained by L-type channels during the plateau phase of the AP. $\mathrm{Ca}^{2+}$ activates RyR2, the ryanodine receptor expressed in cardiac and smooth muscle ${ }^{[7]}$. In skeletal muscle, $\mathrm{Ca}^{2+}$ release is initiated by the surface membrane AP, which spreads via the transverse tubular (t-tubular) system within the fiber. Depolarization of the tubular membrane activates the L-type $\mathrm{Ca}^{2+}$ channel leading to a fast conformational change of one or more intracellular loops which open RyR1, the ryanodine transmission between the t-tubular and sarcoplasmic reticulum membrane is referred to as excitation-contraction coupling receptor expressed in skeletal muscle. Note that the dihydropyridine receptors are voltagegated, while ryanodine receptors are ligand-gated [24].

Mutations in RyR1 are associated with complications related to inhalation anesthetics as well as skeletal muscle myopathies. Dantrolene is a RyR channel blocker and is used to counter the effects of anesthetic-induced malignant hyperthermia. Conversely mutations in RyR 1 that reduce $\mathrm{Ca}^{2+}$ release from the sarcoplasmic reticulum lead to muscle weakness. Dysfunction of RyR2 has been associated with cardiomyopathies. These include a potentially life-threatening arrhythmia due to catecholamine release induced by stressful situations (catecholaminergic polymorphic ventricular tachycardia) ${ }^{[25]}$.

\section{Ion channels with other gating}

Other gating includes activation/inactivation by second messengers from the within of the cell membrane, rather as from outside, as within the case for ligands. Ions may count to such second messengers and then causes direct activation, instead of indirect, as within the case were the electrical potential of ions cause activation/inactivation of voltage-gated ion channels. They include (I) transient receptor potential, (II) cyclic nucleotide-gated channels, (III) ATP sensitive $\mathrm{K}+$ channels, (IV) calcium-activated $\mathrm{K}+$ channels, (V) Mechanosensitive ion channels, (IV) acid-sensing ion channels and (IIV) CF transmembrane ${ }^{[26]}$.

\subsection{Transient receptor potential (TRP) channels}

Some TRP channels seem to be constitutively leaked, while others are gated by voltage, $\mathrm{Ca}^{2+}$ ligand, $\mathrm{pH}$, osmolarity, and mechanical stretch. These channels also vary consistent with the ion(s) they pass, some being selective for $\mathrm{Ca}^{2+}$ while others are less selective, acting as cation channels [27].

The TRP channels play roles during a diverse number of physiological processes which include sensation; including vision, olfaction, mechanosensation, temperature sensation and nocioception, control of bladder function, skin physiology, and respiration. Also, they are targets for the development of drugs to treat pain, epilepsy, anxiety and cancer due to their function (involvement in $\mathrm{Ca}^{2+}$ signaling, sensors for the outside and inside environment of the cell) ${ }^{[18]}$.

Mutations of TRP channels cause various inherited diseases in the cardiovascular, renal, skeletal, and nervous systems ${ }^{[2]}$. 


\subsection{Cyclic nucleotide-gated channels}

They can be divided into two groups: cyclic nucleotide-gated (CNG) and hyperpolarizationactivated cyclic nucleotide-gated (HCN) channels. $\mathrm{HCV}$ is a voltage-gated channel. $\mathrm{CNG}$ is characterized by activation due to the binding of intracellular cAMP or cGMP, with specificity varying by the member. These channels are primarily permeable to $\mathrm{K}^{+}$and $\mathrm{Na}^{+}$. They are also permeable to $\mathrm{Ca}^{2+}$, though it acts to close them ${ }^{[28]}$.

Cyclic GMP binds to the CNG channels allowing $\mathrm{Na}^{+}$and $\mathrm{Ca}^{2+}$ ions to influx and $\mathrm{K}+$ ions to efflux. The inner segments of the rods continually pump $\mathrm{Na}+$ from inside to outside $\left(\mathrm{Na}^{+}-\mathrm{K}^{+}\right.$pump). $\mathrm{Na}+$ will diffuse back through $\mathrm{Na}$ channels to the within (=dark current) to neutralize much of the negativity inside the cell to succeed in-40 $\mathrm{mV}$. These $\mathrm{Na}^{+}$channels are maintained open within the dark by the action of cGMP on $\mathrm{Na}^{+}$channel proteins. This dark current depolarizes the photoreceptor. The passive spread of this depolarization from the outer segment (where the $\mathrm{Na}^{+}$channels are located) to the synaptic terminal (where the photoreceptor's neurotransmitter is stored) opens the voltage-gated $\mathrm{Ca}^{2+}$ channels and causes the discharge of the neurotransmitter glutamate from the synaptic terminal while within the dark. Glutamate can then stimulate receptors on second-order visual neurons ${ }^{[29]}$.

On exposure to light, the rhodopsin is activated rapidly into the active form - metarhodopsin II and catalyzes the exchange of GTP for GDP bound to a $\mathrm{G}$ protein called transducin. The GTP-bound form of transducin is active and stimulates a cGMPphosphodiesterase to increase the hydrolysis of cGMP. The consequent decrease in cytoplasmic cGMP concentration leads to the closure of the cGMP-activated channels and the back diffusion is stopped while the $\mathrm{Na}^{+}$pump continues which leads to hyperpolarization $(-70 \mathrm{Mv})$. This light-induced hyperpolarization reduces or stops the release of glutamate from the photoreceptor to second-order visual neurons. The phototransduction mechanism in cones is similar, except for quantitative differences such as lower light sensitivity and faster response kinetics in these cells ${ }^{[28]}$.

CNG channels also are involved in olfactory transduction within the cilia of vertebrate olfactory receptor neurons. Odor substances bind with odorant-binding protein present in olfactory mucosa that increase the odor concentration and transport them to receptors. These activate $\mathrm{G}$ protein that activates adenyl cyclase and form cyclic AMP. The cyclic AMP causes the opening of $\mathrm{CNG} \mathrm{Na}^{+}$ channels resulting in the influx of $\mathrm{Na}+$ and generation of the receptor potential. The olfactory cyclic CNG channel has properties rather almost like those of the rod and cone cGMP-activated channels, except that it requires a way lower cyclic nucleotide concentration to open ${ }^{[26]}$.

Mutations in CNG genes have been shown to cause achromatopsia, a hereditary visual disorder, which is characterized by decreased vision, light sensitivity, and the absence of color vision ${ }^{[2]}$.

\subsection{ATP sensitive $\mathrm{K}^{+}$channels (IKATP)}

It occurs at both the sarcolemmal and mitochondrial inner membranes of cardiomyocytes. It's inhibited by physiological intracellular ATP and opens when ATP decreases during cellular hypoxia. Thus, under normal energetic circumstances, there's limited IKATP current. However, under both physiological and pathological conditions that reduce ATP, there's increased IKATP current that's essential for adaptation to stress ${ }^{[2]}$.

In the heart, in response to ischemic stress, they play a protective role and in vascular smooth muscle regulation of vascular to (vasodilatation). In the brain, including the substantia nigra and within the hypothalamus KATP channels may play a task within the suppression of seizures in ATP depleted conditions. They are the main drug target (diabetes, angina, severe hypertension, and baldness) among $\mathrm{K}^{+}$channels ${ }^{[30]}$.

In pancreatic $\beta$-cells, a rise in ATP/ADP ratio, which is generated by glucose uptake and metabolism, closes the IKATP channels to elicit membrane depolarization, $\mathrm{Ca}^{2+}$ influx, and secretion of insulin, the first hormone of glucose homeostasis. Thus, IKATP channels are implicated in hypoglycemia, type II DM, and smooth muscle relaxation ${ }^{[10]}$.

\subsection{Calcium activated $\mathrm{K}^{+}$channels $(\mathrm{KCa})$}

They respond to changes in the intracellular $\mathrm{Ca}^{2+}$ ion concentration. When activated they allow $\mathrm{K}^{+}$ions to efflux to either repolarize or 
hyperpolarize the cell membrane. This causes $\mathrm{Ca} 2+$ channels to become deactivated (and stimulates the $\mathrm{Na}^{+} / \mathrm{Ca}^{2+}$ exchanger to pump $\mathrm{Ca}^{2+}$ ions out of the cytosol) thereby limiting the intracellular concentration of $\mathrm{Ca}^{2+}$ ions. Therefore $\mathrm{KCa}$ channels play a role in determining the amplitude and duration of $\mathrm{Ca}^{2+}$ transients and the downstream signaling pathways that perturbation in $\mathrm{Ca}^{2+}$ ion concentration influence ${ }^{[31]}$.

\subsection{Mechanosensitive Ion Channels (or stretch- gated ion channels)}

Mechanosensitive ion channels are found in nearly all kinds of cells. They're expressed within the lungs, bladder, skin, red blood cells, and vascular cells. They're the sensors for several systems including the senses of touch, vision, hearing, taste, smell, heat sensation, and balance, also as participating in cardiovascular regulation and osmotic homeostasis ${ }^{[2]}$.

There are two mammalian mechanosensitive ion channels, namely Piezo1 and Piezo2. The best example is hair cells involved within the hearing process. The upper end of the hair cell is ciliated. Each hair cell has small freely-movable cilia called stereocilia and one large stiff cilium called the kinocilium. When a wave of sound deflects the stereocilia, the $\mathrm{K}^{+}$channel opens. $\mathrm{K}^{+}$ enters the cells and hair cells depolarize (cochlear microphonic potential). Epithelial cell depolarization opens voltage-gated $\mathrm{Ca}^{2+}$ channels causing the discharge of the excitatory neurotransmitters (glutamate) that stimulate the cochlear nerve then to the cortex ${ }^{[32]}$.

Stretch-activated ion channels are correlated with major pathologies; include arrhythmia (such as atrial fibrillation), cardiac hypertrophy, Duchenne dystrophy and other cardiovascular diseases. Mutations of Piezol cause hereditary xerocytosis and autosomal dominant hemolytic anemia. This is often while, Piezo2 is expressed in sensory neurons of the dorsal root and trigeminal ganglia, suggesting it plays a task within the sensation of touch. Distal arthrogryposis, a disorder characterized by deformities in joints (contractures) restricting hand and feet movement are examples of these channel mutations ${ }^{[33]}$.

\subsection{Acid-Sensing Ion Channels (ASIC)}

They are widely expressed within the central, and peripheral nervous systems. Because ASICs are permeable to $\mathrm{Na}^{+}$and $\mathrm{Ca}^{2+}$, their activation results in membrane depolarization ${ }^{[7]}$. They're involved in important neurological diseases and conditions, like pain sensation, fear, synaptic function, plasticity, anxiety, epilepsy, and neurodegeneration after ischemia. Neuronal degeneration and death during pathological extracellular acidosis caused by ischemia, autoimmune inflammation, and traumatic injury can be induced by this channel ${ }^{[34]}$.

\subsection{Cystic Fibrosis Transmembrane Conductance Regulator $\mathrm{Cl}^{-}$Channels(CFTR)}

This channel is transport $\mathrm{Cl}^{-}$in and out of cells, controlling water movement in tissues, a necessity for the assembly of mucus that lubricates and protects the liner of the airways, digestive and reproductive systems and other organs [2]. CFTR expression is abundant within the human kidney. It is found right along the nephron, including proximal and distal tubules, thin and thick ascending limbs, and therefore the collecting duct. Dysfunction of those channels results in many diseases, including cystic fibrosis and bronchiectasis ${ }^{[35]}$.

\section{References}

1 Bezanilla F. Voltage-gated ion channels. IEEE Trans Nanobioscience 2005; 4(1): 34-48 DOI: 10.1109/tnb.2004.842463

2 Rubaiy HN. A Short Guide to Electrophysiology and Ion Channels. J Pharm Pharm Sci 2017; 20: 48-67 [PMID: 28459656 DOI: $10.18433 / \mathrm{J} 32 \mathrm{P} 6 \mathrm{R}]$

3 Berridge MJ. The Inositol Trisphosphate/Calcium Signaling Pathway in Health and Disease. Physiol Rev 2016; 96(4): 1261-1296 DOI: 10.1152/physrev.00006.2016

4 Zaydman MA, Silva JR, Cui J. Ion Channel Associated Diseases: Overview of Molecular Mechanisms. Chemical

Reviews 2012; $\quad$ 112(12): 6319-6333.

DOI: $10.1021 / \mathrm{cr} 300360 \mathrm{k}$

5 Abdul Kadir L, Stacey M, Barrett-Jolley R. Emerging Roles of the Membrane Potential: 
Action Beyond the Action Potential. Frontiers in Physiology 2018; 9: 1661 .

DOI:10.3389/fphys.2018.01661

6 Nishida H, Matsumoto A, Tomono N, Hanakai T, Harada S, Nakaya H. Biochemistry and Physiology of Mitochondrial Ion Channels Involved in Cardioprotection. FEBS letters 2010; 584(10): 2161-2166. [PMID: 20035754. DOI:10.1016/j.febslet.2009.12.033]

7 Alexander S, Mathie A, Peters JA, Veale EL, Striessnig J, Kelly E, Armstrong JF, Faccenda E, Harding SD, Pawson AJ, Sharman JL, Southan C, Davies JA, CGTP Collaborators. The Concise Guide to Pharmacology 2019/20: Ion Channels. British Journal of Pharmacology 2019; 176 (1): S142 - S228. . DOI:10.1111/bph.14749

8 Baenziger JE, Corringer PJ. 3D Structure and Allosteric Modulation of The Transmembrane Domain of Pentameric Ligand-Gated Ion Channels. Neuropharmacology 2011, 60(1): 116-125. [PMID: 20713066. DOI:10.1016/j.neuropharm.2010.08.007]

9 Gotti C, Clementi F, Fornari A, Gaimarri A, Guiducci S, Manfredi I, Moretti M, Pedrazzi P, Pucci L, Zoli M. Structural and Functional Diversity of Native Brain Neuronal Nicotinic Receptors. Biochemical Pharmacology 2009; 78(7): 703 - 711. [PMID: 19481063. DOI:10.1016/j.bcp.2009.05.024]

10 Lehmann-Horn F, Jurkat-Rott K. VoltageGated Ion Channels and Hereditary Disease. Physiological Reviews 1999; 79(4): 1317-1372. [PMID: 10508236. DOI:10.1152/physrev.1999.79.4.1317]

11 Fakhfouri G,Mousavizadeh K, Mehr SE, Dehpour AR, Zirak MR, Ghia JE, Rahimian R. From Chemotherapy-Induced Emesis to Neuroprotection: Therapeutic Opportunities For 5-ht3 Receptor Antagonists. Molecular Neurobiology 2015; 52(3), 1670-1679. [PMID: 25377794. DOI:10.1007/s12035-014-8957-5]

12 Peralta FA, Huidobro-Toro JP. Zinc as Allosteric Ion Channel Modulator: Ionotropic Receptors as Metalloproteins. International Journal of Molecular Sciences 2016; 17(7): 1059. DOI:10.3390/ijms17071059

13 Davies PA, Wang W, Hales TG, Kirkness EF. A Novel Class of Ligand-Gated Ion Channel is
Activated by Zn2+. The Journal of Biological Chemistry 2003; 278(2): 712 - 717. [PMID: 12381728. DOI:10.1074/jbc.M208814200]

14 Vithlani M,Terunuma M, Moss SJ. The Dynamic Modulation of GABA (A) Receptor Trafficking and Its Role in Regulating The Plasticity of Inhibitory Synapses. Physiological Reviews 2011; 91(3): 1009-1022. DOI:10.1152/physrev.00015.2010

15 Mele M,Leal G, Duarte CB. Role of GABAA R Trafficking in The Plasticity of Inhibitory Synapses. Journal of Neurochemistry 2016; 139(6): 997-1018. [PMID: 27424566. DOI:10.1111/jnc.13742]

16 Sigel E, Steinmann ME. Structure, Function, and Modulation of GABA(A) Receptors. The Journal of Biological Chemistry 2012; $\quad 287(48)$ : 40224-40231.

DOI:10.1074/jbc.R112.386664

17 Kaczorowski GJ, McManus OB, Priest BT, Garcia ML. Ion Channels As Drug Targets: The Next GPCRs. The Journal of General Physiology 2008; 131(5): 399-405. DOI:10.1085/jgp.200709946

18 Lynch JW. Native Glycine Receptor Subtypes and Their Physiological Roles. Neuropharmacology 2009; 56: 303-309. DOI:10.1016/j.neuropharm.2008.07.034

19 Hansen KB, Yi F, Perszyk RE, Furukawa H, Wollmuth LP, Gibb AJ, Traynelis S F. Structural Insights Into The Mechanisms of CNBD Channel Function. The Journal of General Physiology 2018; 150(8): 1081-1105. . DOI:10.1085/jgp.201812032

20 Twomey EC, Yelshanskaya MV,Sobolevsky AI. Structural and Functional Insights Into Transmembrane AMPA Receptor Regulatory Protein Complexes. The Journal of General Physiology 2019; 151(12): 1347-1356. DOI:10.1085/igp.201812264

21 Habermacher C, Dunning K, Chataigneau T, Grutter T. Molecular Structure and Function of P2X Receptors. Neuropharmacology 2016; 104: 18-30. [PMID: 26231831. DOI:10.1016/j.neuropharm.2015.07.032]

22 Hamada K , Mikoshiba K . IP3 Receptor Plasticity Underlying Diverse Functions. Annual Review of Physiology 
2020; 82: 151-176. [PMID: 31730387. DOI:10.1146/annurev-physiol-021119-034433]

23 Mikoshiba K. Role of IP3 Receptor Signaling in Cell Functions and Diseases. Advances in Biological Regulation 2015; 57: 217-227. DOI:10.1016/j.jbior.2014.10.001

24 Meissner G. The Structural Basis of Ryanodine Receptor Ion Channel Function. The Journal of General Physiology 2017; 149(12): 10651089. DOI: 10.1085 /jgp.201711878

25 Jiang D, Chen W, Xiao J, Wang R, Kong H, Jones PP, Zhang L, Fruen B, Chen SR. Reduced Threshold for Luminal $\mathrm{Ca} 2+$ Activation of RyR1 Underlies a Causal Mechanism of Porcine Malignant Hyperthermia. The Journal of Biological Chemistry 2008; 283(30): 20813-20820. DOI:10.1074/jbc.M801944200

26 Hanukoglu I. ASIC and ENaC type sodium channels: conformational states and the structures of the ion selectivity filters. FEBS J. 2017; 284: 525-545 [PMID:27580245]

27 Cao E. Structural Mechanisms of Transient Receptor Potential Ion Channels. The Journal of General Physiology 2020;152(3): e201811998. DOI:10.1085/jgp.201811998

28 Biel M, Michalakis S. Cyclic Nucleotide-Gated Channels. Handb Exp Pharmacol. 2009; (191):111-136. [PMID: 19089328. DOI:10.1007/978-3-540-68964-5 7]

29 Mazzolini M,Arcangeletti M, Marchesi A, Napolitano L, Grosa D, Maity S, Anselmi C, Torre V. The Gating Mechanism in Cyclic Nucleotide-Gated Ion Channels. Scientific Reports 2018; 8(1): 45. DOI:10.1038/s41598$\underline{017-18499-0}$
30 Sun HS, Feng ZP. Neuroprotective Role of ATP-Sensitive Potassium Channels in Cerebral Ischemia. Acta Pharmacologica Sinica 2013; 34(1): 24-32.

DOI: 10.1038/aps.2012.138

31 Weaver AK, Bomben VC, Sontheimer H. Expression and Function of Calcium-Activated Potassium Channels in Human Glioma Cells. Glia 2006; 54(3): 223-233. DOI:10.1002/glia.20364

32 Martinac B. Mechanosensitive Ion Channels: An Evolutionary and Scientific Tour De Force in Mechanobiology. Channels (Austin, Tex.) 2012; 6(4): 211-213. DOI: $10.4161 /$ chan.22047

33 Ranade SS, Syeda R, Patapoutian A. Mechanically Activated Ion Channels. Neuron 2015; 87(6): 1162-1179. DOI:10.1016/j.neuron.2015.08.032

34 Osmakov DI, Andreev YA, Kozlov SA. AcidSensing Ion Channels and Their Modulators. Biochemistry. Biokhimiia 2014; 79(13): 1528-1545. [PMID: 25749163. DOI:10.1134/S0006297914130069]

35 Hwang TC, Yeh JT, Zhang J, Yu YC, Yeh HI, Destefano S. Structural Mechanisms of CFTR Function and Dysfunction. The Journal of General Physiology 2018; 150(4): 539570. DOI:10.1085/jgp.201711946 